\title{
OPENING RANGE BREAKOUT STOCK TRADING ALGORITHMIC MODEL
}

\author{
Mrs.S.Mahalakshmi ${ }^{1}$ and Mr.Vignesh $\mathrm{P}^{2}$ \\ ${ }^{1}$ Assistant Professor, Department of ISE, BMSIT\&M, Bengaluru, India \\ ${ }^{2}$ Student,Department of ISE, BMSIT\&M, Bengaluru, India
}

\begin{abstract}
Stock Trading Algorithmic Model is an important research problem that is dealt with knowledge in fundamental and technical analysis, combined with the knowledge expertise in programming and computer science. There have been numerous attempts in predicting stock trends, we aim to predict it with least amount of computation and to decrease the space complexity. The goal of this paper is to create a hybrid recommendation system that will inform the trader about the future of a stock trend in order to improve the profitability of a short term investment. We make use of technical analysis tools to incorporate this recommendation into our system. In order to understand the results, we implemented a prototype in $R$ programming language.
\end{abstract}

\section{KEYWORDS}

Stock Trading Algorithmic Model, Breakout Trading, R Programming, Short term trading.

\section{INTRODUCTION}

Stock Trading Algorithms have gained a significant place in research due to its profitable benefits. A lot of researches are focused to find solutions on how to make a good investment. From the survey we have concluded that there are multiple solutions in order to acquire results. Some of these solutions are: By using fundamental analysis and technical analysis [1], Arbitrage analysis [1], Time series model [1], Temporal Data mining or by combining them to have more precise prediction [10].

The main problems are that it is not easy to predict the Stock Market trend because of its non linear, dynamic and chaotic identity, combined with macroeconomic factors and noisy time series. To forecast, technical analysis uses factors such as charts, market indicators, and the relationship between volume and price indicators. In Technical Analysis, there are multiple techniques such as gap analysis, breakout system, market models, momentum precedes price and neural networks.

Artificial intelligence has gained a significant amount of importance in predicting stock trends. Techniques in Artificial intelligence such as neural network are used in order to capture patterns, relationships and environmental changes based on historical data and determine future trends of a stock. We have developed an environment that will be able to combine technical analysis tools and provide a blackboard based architecture based on the literature survey. 


\section{RELATED WORK}

Andrea.S et.al proposed that a good prediction can be done by using macroeconomic variables, time series combination and temporal data mining [1]. It can also be done by combining fundamental analysis and technical analysis, with a multi agent system.[2][3]. D.Maldovan [4] uses macroeconomic variables because a change in the rule should be considered as a random event and not predictable.

The most popular approach in recent times is predicting stock using Neural Networks Models for good estimation of a non-linear function without knowing the characteristics of the data [4], [5], [6] and Markov Regime Switching Models due to the fact that it can capture discrete shifts which can appear in the behaviour of economic variables [5] for forecasting financial time series.

Numerous approaches have been used in the past to create expert systems which deal with stock forecasting by using fundamental and technical analysis such as MASST (Multi-Agent System for Stock Trading) [7] which was designed to be an agent-based environment able to collect, filter and manage data in order to make suggestions to the user. The architecture of this system lies on three agents which are interconnected.The agents were Location agent, stock sample agent and symbol agent.

G. Preethi has proposed a trading algorithmic model that uses the signal of three technical indicators to produce a trading signal. These technical indicators are MACD (Moving Average Convergence-Divergence), ROC (Price Rate of Change) and STS (Stochastic Oscillator) [8].

G.Marketos et.al have worked on "Intelligent Stock Market Assistant using Temporal data mining". He discuss about portfolio management solutions using business intelligence by the use of temporal data mining patterns.

\section{Motivation}

The motivation behind the paper is to find the most efficient method of prediction with less computation and utilizing fewer system resources. The goal is to forecast the stock market based on the first 45 minutes of the day's data. This method is quite famously known as Opening Range Breakout. Based on research conducted on ORB trading it gives a better prediction result than those based on historical data. We convert this technique into an algorithm form and make it an Algorithmic Trading Platform so that the entire process of calculating would be done in a matter of seconds.

\section{Problem Statement}

One of the major problems faced in modelling financial market movements is the fact that information comes in from a very large number of sources. With many models developed in this field, we decided to take an approach away from such large data analysis (historical data). The challenge here is to develop a model that has a good performance and best accuracy. While taking very less amount of data analysis, the end goal is to find the best configuration for the model to give the maximum performance. In our project, we are considering 50 stocks in all the twelve sectors of Nifty, an index composed by National Stock Exchange of India. In our model, we are aiming to achieve an accuracy of approximately $60-70 \%$. 


\section{Proposed Methodology}

Traditional Stock Market analysis mainly depends on data. Different techniques such as artificial neural network, genetic algorithm and so on depends on historical data. The main problem with historical data is that sometimes the data on some particular day won't be available and also the data will be very large. Our project forecasts the stock market based on the first 45 minutes of the opening data. This method is popularly known as Opening Range Trading. Based on the research conducted, Opening Range Breakout trading gives better results than those predictions which are based on the historical data.

We convert this technique into an algorithm form and make it as an algorithmic trading platform. So that the entire process of calculation would be done in a matter of seconds and by adding customized filters to the algorithm increases the efficiency, compared to conventional method, thereby increases the chances of high profitability. The main objective of this project is to determine the right opportunity to invest in a particular stock, to maximize the profit and minimize the loss.

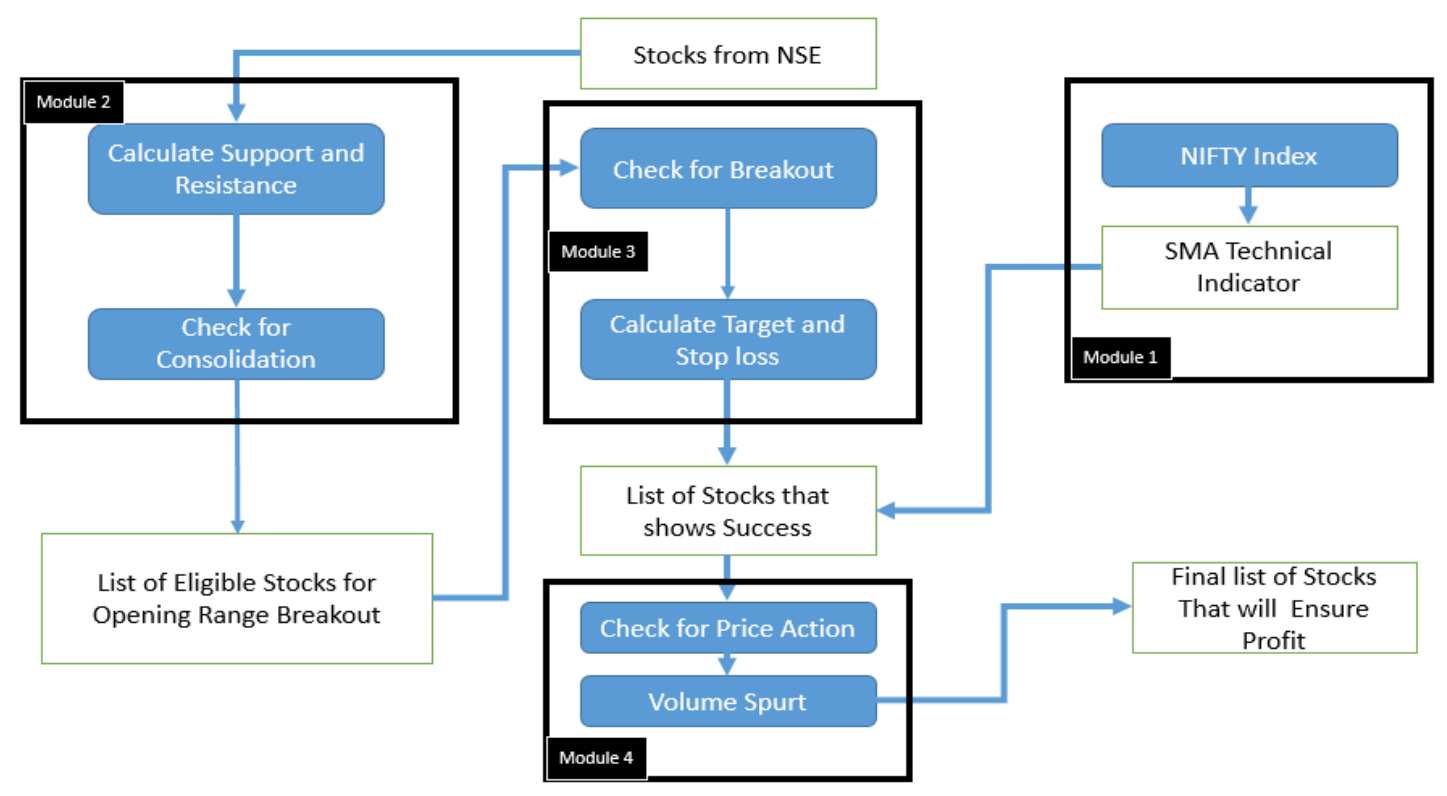

Fig 1 - Methodology

\section{IMPLEMENTATION}

The solution has been divided into modules in order to implement. Our data source for the project is Google finance that provides real-time data for the application

\subsection{Simple Moving Average}

Access the Nifty 50 and plot simple moving average on the accessed 50 stocks of nifty. A simple moving average is calculated by adding the closing price of the stock for a number of time periods and then dividing this total by the number of time periods. We consider two moving averages - a short period and a long period. If the shorter period, crosses and goes above the longer period, we know that the market is going up but if the opposite happens, we come to know that the market is going down. The relationship between shorter and longer moving average trend lines is the subject of many studies and traders look to crossovers for opportunities to buy, sell or short. 


\subsection{Calculation of Support and Resistance}

Scans the accessed 50 stocks and finds out support and resistance based on the first thirty minutes and also check if support and resistance are broken or not during the consolidation period. If consolidation period is not broken then stocks are eligible for trading and rest of the stocks are eliminated for the day. The support and resistance lines are set by observing the time between 9:25 a.m. and 9:55 a.m. [9]. The high and low for that period is set as the support and resistance.

\subsection{Setting the Buying Price, Target, Stop Loss}

Post consolidation period, the program checks if the support and resistance are broken and if it has, then the buying price, target and stop loss is printed. The target is calculated by computing the difference between the support and resistance and the dividing that by 2 . The stop loss is calculated by dividing the difference by 3 . The objective of setting the target is to ensure that the investor gets a fair share of the market's profit. The purpose of the stop loss is to guarantee a failsafe method of losing money. The investor does lose money, but at minimum risk.

\subsection{Monitoring the Price Action}

Once the Breakout occurs, it checks if the breakout occurred is a false breakout or true breakout by comparing the breakout bar with the next bar. We use an active price filter and a volume spurt filter. If the first breakout bar is bullish, the second bar needs to be bullish too. Otherwise, there is a chance of the market going down and the investor losing his money. The same applies for a bearish bar too. If this pattern isn't followed, it means that it is a false breakout and the target will not be reached. Sequence diagram described in fig [2] will show the order of execution of the modules.

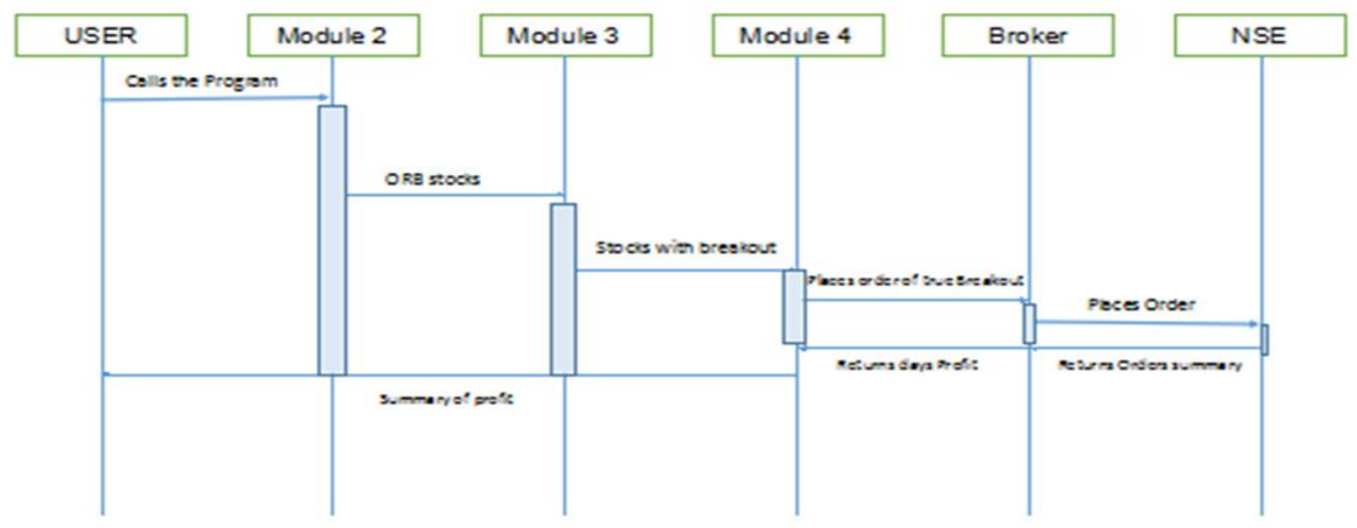

Fig 2. Sequence Diagram

\section{TESTING}

\subsection{Accuracy Testing Results}

The algorithm was tested for a period of 7 trading days from 25 Mar 2015 to 02 Apr 2015. This period was chosen randomly. On an average 25 trades were done on each day, of which a 
prediction accuracy of $70 \%$ was achieved by testing the algorithm in the real-time market. The results are tabulated in the table [1].

\subsection{Single Day Real Time Test Results}

A single random day test was carried out on 19-05-2015 (Tuesday) to test the performance of the algorithm. The following was observed and recorded in table [2]. It was noted that stocks in the automobile, banks, and steel companies achieved the target. On the other hand, cement and oil companies failed to achieve the target and few were with no trade.

Table1. Prediction Accuracy Percentage

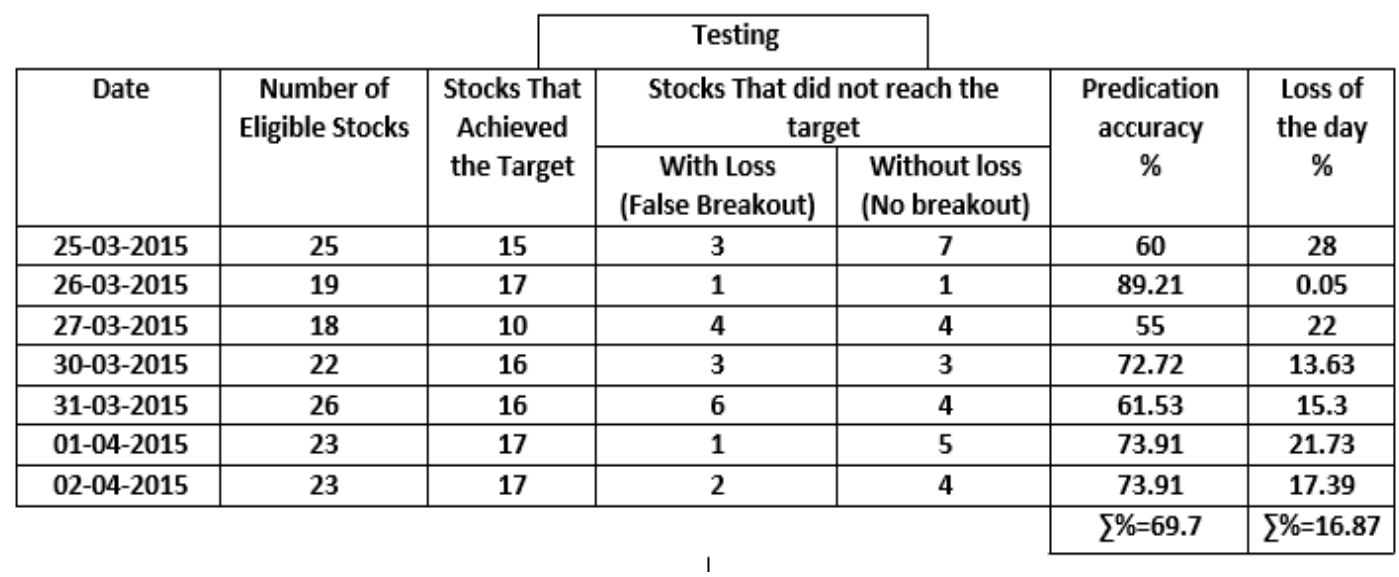

Table 2. Single Day Real Time Test Results

\begin{tabular}{|c|c|c|c|c|c|c|c|}
\hline \multirow[t]{2}{*}{ Date } & \multirow[t]{2}{*}{$\begin{array}{l}\text { List of Eligible } \\
\text { Stocks }\end{array}$} & \multirow{2}{*}{$\begin{array}{c}\text { Stock } \\
\text { Achieved } \\
\text { the } \\
\text { Target }\end{array}$} & \multicolumn{2}{|c|}{$\begin{array}{c}\text { Stocks That did not reach } \\
\text { the target }\end{array}$} & \multirow[t]{2}{*}{ BUY/SELL } & \multirow{2}{*}{$\begin{array}{c}\text { Prediction } \\
\text { accuracy } \\
\not \%\end{array}$} & \multirow{2}{*}{$\begin{array}{c}\text { Loss of the } \\
\text { day } \\
\%\end{array}$} \\
\hline & & & $\begin{array}{l}\text { With Loss } \\
\text { (False } \\
\text { Breakout) }\end{array}$ & $\begin{array}{c}\text { Without loss } \\
\text { (No } \\
\text { breakout) }\end{array}$ & & & \\
\hline \multirow{25}{*}{ 19-05-2015 } & $\mathrm{ACC}$ & - & $\checkmark$ & - & - & \multirow{25}{*}{60} & \multirow{25}{*}{28} \\
\hline & AMBUJACEM & $\checkmark$ & - & - & Sell->Buy & & \\
\hline & ASIANPAINT & $\checkmark$ & - & - & Sell->Buy & & \\
\hline & AXISBNAK & $\checkmark$ & - & - & Buy->Sell & & \\
\hline & BAJAJ-AUTO & - & - & $\checkmark$ & - & & \\
\hline & BHARTIARTL & $\checkmark$ & - & - & Buy->Sell & & \\
\hline & CAIRN & $\checkmark$ & - & - & Buy->Sell & & \\
\hline & CIPLA & - & $\checkmark$ & - & - & & \\
\hline & DRREDY & $\checkmark$ & - & - & Buy->Sell & & \\
\hline & GAIL & - & - & $\checkmark$ & - & & \\
\hline & HINDUNILVR & $\checkmark$ & - & - & Sell->Buy & & \\
\hline & IDFC & $\checkmark$ & - & - & Sell->Buy & & \\
\hline & INFY & $\checkmark$ & - & - & Sell->Buy & & \\
\hline & ITC & - & $\checkmark$ & - & - & & \\
\hline & JINDALSTEL & $\checkmark$ & - & - & Sell->Buy & & \\
\hline & LT & $\checkmark$ & - & - & Sell->Buy & & \\
\hline & MARUTI & $\checkmark$ & - & - & Buy->Sell & & \\
\hline & NMDC & - & $\checkmark$ & - & - & & \\
\hline & ONGC & - & $\checkmark$ & - & - & & \\
\hline & POWERGRID & - & $\checkmark$ & - & - & & \\
\hline & RELIANCE & - & - & $\checkmark$ & - & & \\
\hline & SBIN & $\checkmark$ & - & - & Sell->Buy & & \\
\hline & SSLT & $\checkmark$ & - & - & Buy->Sell & & \\
\hline & TATAMOTERS & - & $\checkmark$ & - & - & & \\
\hline & WIPRO & $\checkmark$ & - & - & Buy->Sell & & \\
\hline & & & & & & 60 & 28 \\
\hline
\end{tabular}




\section{CONCLUSION}

The Opening Range is a powerful concept and yields significant profits when traded with the right stock. It works best with a consolidation period of 25 minutes, an active price filter, and volume spurt filter. Sometimes the market is very volatile due to external influences like politics, company mergers etc., therefore on those days it is difficult to have a high prediction accuracy. Overall, we are able to achieve an accuracy of almost $70 \%$. In future, we can automate this process by linking it with iBroker, which is an interactive broker system to make it completely automated.

\section{SCREENSHOT'S}

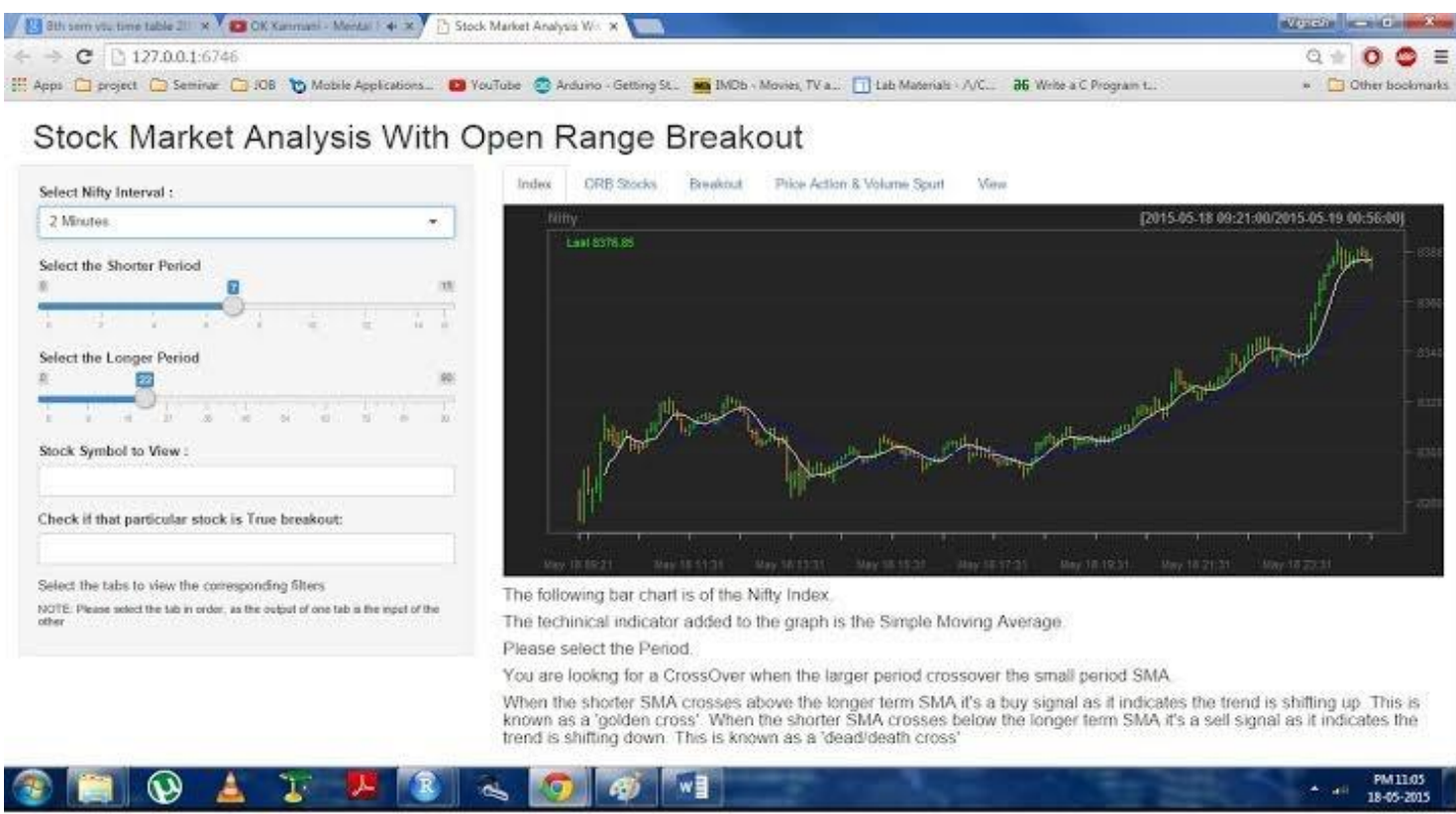

Fig 3. SMA

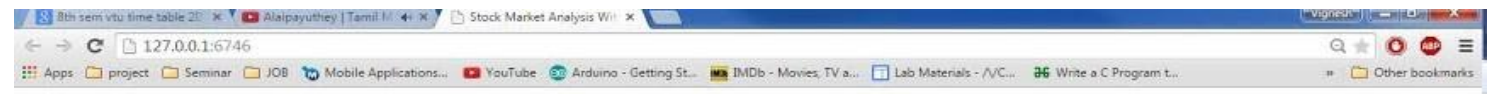

Stock Market Analysis With Open Range Breakout
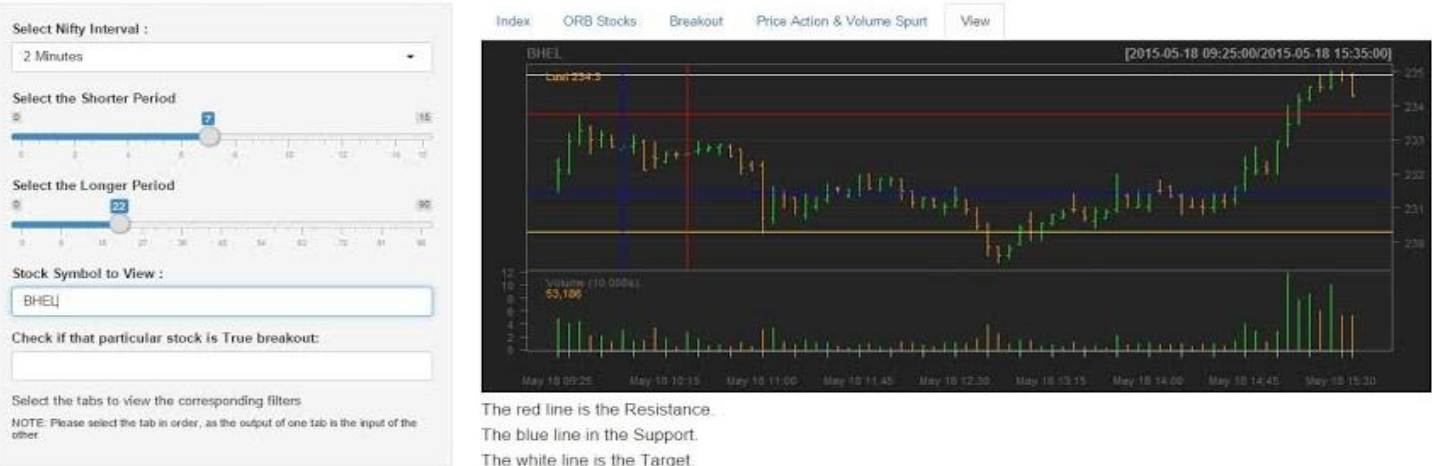

The red line is the Resistance

The blue line in the Support

The white line is the Targe

Fig 4. Breakout 


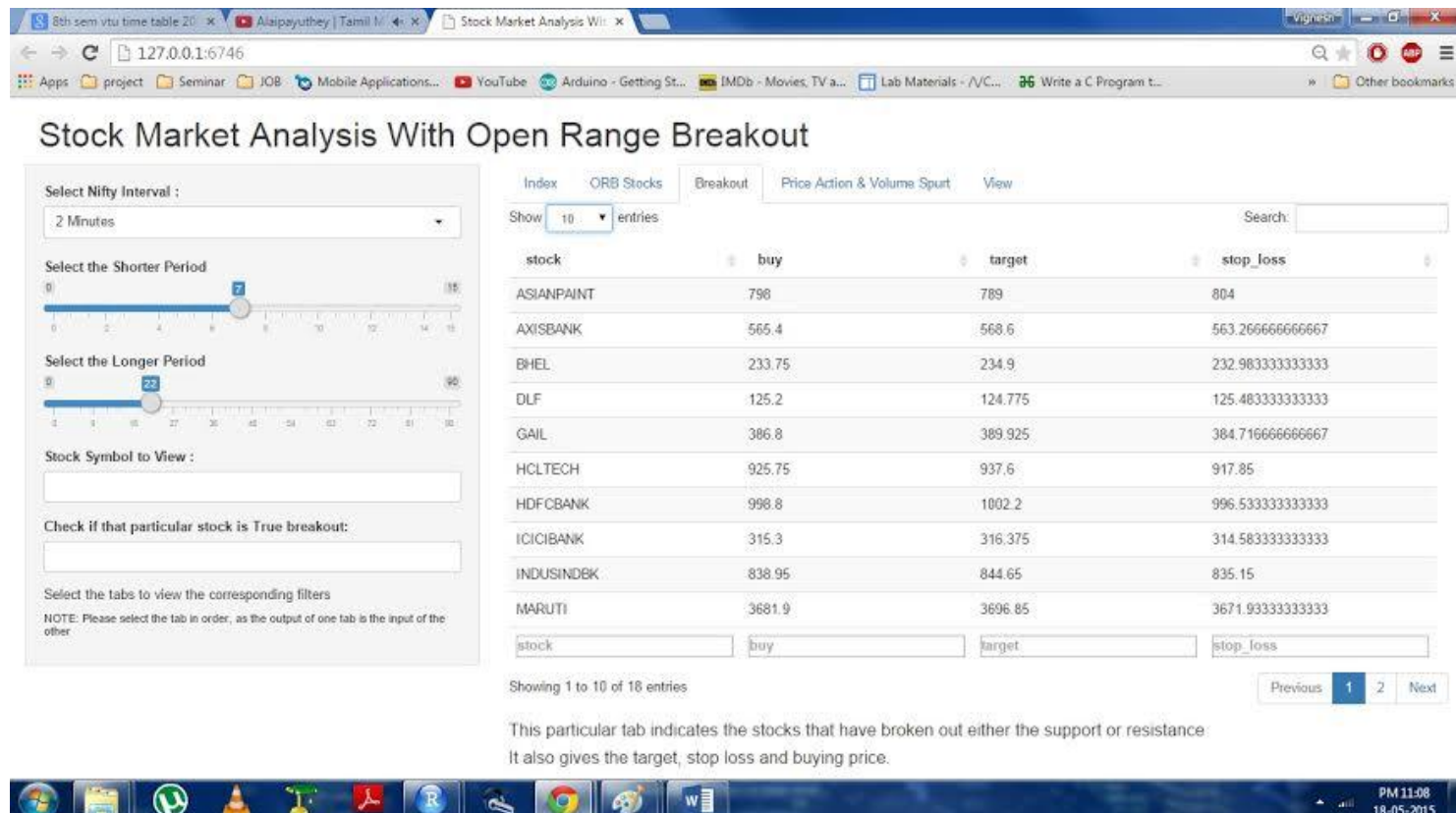

Fig 5. Buying Price, Target, Stop Loss

\section{REFERENCES}

[1] Andreas S. Andreou, Constantinos C. Neocleous, Christos N. Schizas, "Testing the predictability of the Cyprus Stock Exchange :The case of an emerging market in Proceedings of the IEEE-INNSENNS International Joint Conference on Neural Networks, Vol:6,2000

[2] Andrew Pole, Statistical Arbitrage: Algorithmic Trading Insights and Techniques, John Wiley \& Sons, 2007

[3] Monica Tirea, Viorel Negru, "Stock Market Analysis - Strongest Performing Stocks Influence on an Evolutionary Market" in Proceedings of the 35th International Conference on Information Technology Interfaces (ITI), 2013

[4] D. Moldovan, M. Moca, t. NitchiI, “A Stock Trading Algorithm Model Proposal, based on Technical Indicators Signals", Informatica Economic, Vol 15, Issue No 1, pp.183-188, 2011

[5] Fluctuations Gabriel Iuhasz, Monica Tirea and Viorel Negru, "Neural Network Predictions of Stock Price", Published in 14th International Symposium on Symbolic and Numeric Algorithms for Scientific Computing (SYNASC), 2012

[6] C. Xiang and W. M. Fu, "Predicting the Stock Market using Multiple Models", in 9th International Conference on Control, Automation, Robotics and Vision, 2006.

[7] D. Liu, L. Zhang, "China Stock Market Regime Prediction with Artificial Neural Network and Markov Regime Switching”, in Proceedings of the World Congress on Engineering, Volume 1, 2010, London.

[8] G.Preethi, B. Santhi, "Stock Market Forecasting Technique: a Survey”, Journal of Theoretical and Applied Information Technology, Vol. 46 ,pp:No.1 , 2012.

[9] http://www.marketgauge.com/ TradingThe10OclockBulls Winning Strategies for Active Traders by Geoff Bysshe

[10] Wei Yang, "Stock Price Prediction Based On Fuzzy Logic", Published in Proceedings of the Sixth International Conference on Machine and Cybernetics, Vol: 3,2007

[11] Marketos et.al, "Intelligent Stock Market Assistant Using Temporal data mining”, published in Proceedings of 10th Panhellenic Conference in Informatics (PCI'05), Volos, Greece, November 2005. 